\title{
Gold nanoparticles attenuates bacterial sepsis in cecal ligation and puncture mouse model through the induction of $\mathrm{M} 2$ macrophage polarization
}

Sujittra Taratummarat ${ }^{1}$, Naunpun Sangphech², Chau Tran Bao Vu ${ }^{3}$, Tanapat Palaga², Thunnicha Ondee ${ }^{4}$, Saowapha Surawut ${ }^{1}$, Amornpun Sereemaspun ${ }^{5}$, Patcharee Ritprajak ${ }^{6}$ and Asada Leelahavanichkul ${ }^{7,8^{*}}$ (D)

\begin{abstract}
Background: Gold nanoparticles (AuNP) have several biochemical advantageous properties especially for a candidate of drug carrier. However, the non-conjugated AuNP has a higher rate of cellular uptake than the conjugated ones. Spherical AuNP in a proper size $(20-30 \mathrm{~nm})$ is non-toxic to mice and shows anti-inflammatory properties. We tested if the administration of AuNP, as an adjuvant to antibiotics, could attenuate bacterial sepsis in cecal ligation and puncture (CLP) mouse model with antibiotic (imipenem/cilastatin).

Results: Indeed, AuNP administration at the time of CLP improved the survival, blood bacterial burdens, kidney function, liver injury and inflammatory cytokines (TNF-a, IL-6, IL-1 $\beta$ and IL-10). AuNP also decreased M1 macrophages (CD86 + ve in F4/80 + ve cells) and increased M2 macrophages (CD206 + ve in F4/80 + ve cells) in the spleens of sepsis mice. The weak antibiotic effect of AuNP was demonstrated as the reduction of E. coli colony after $4 \mathrm{~h}$ incubation. In addition, AuNP altered cytokine production of bone-marrow-derived macrophages including reduced TNF- $a$, IL-6 and IL-1 $\beta$ but increased IL-10 at 6 and 24 h. Moreover, AuNP induced macrophage polarization toward anti-inflammatory responses (M2) as presented by increased Arg1 (Arginase 1) and PPARY with decreased Nos2 (inducible nitric oxide synthase, iNos) and Nur77 at $3 \mathrm{~h}$ after incubation in vitro.

Conclusions: The adjuvant therapy of AuNP, with a proper antibiotic, attenuated CLP-induced bacterial sepsis in mice, at least in part, through the antibiotic effect and the induction of macrophage function toward the anti-inflammatory responses.
\end{abstract}

Keywords: Sepsis, Gold nanoparticles, Cecal ligation and puncture, Macrophage polarization

\section{Background}

Sepsis is a syndrome of organ dysfunction due to dysregulated host responses to systemic infection, independent of the type of organisms [1]. Bacterial sepsis is an important world-wide healthcare problem and it is a major cause of death in patients with clinically ill conditions [1]. The imbalance of pro- and anti-inflammatory

\footnotetext{
* Correspondence: aleelahavanit@gmail.com

7Department of Microbiology, Faculty of Medicine, Chulalongkorn University, Bangkok 10330, Thailand

${ }^{8}$ Center of Excellence in Immunology and Immune-mediated Diseases,

Department of Microbiology, Faculty of Medicine Chulalongkorn University, Bangkok, Thailand

Full list of author information is available at the end of the article
}

immune responses is one of the important sepsis pathophysiology [2]. Both overt- and insufficient- immune responses to sepsis could be harmful to patients [2, 3]. As such, the moribund stage in sepsis could be a result of hyper-immune response or immune-suppressive reaction [4]. Hence, the rapid organism control with a proper immune modulation might be a proper strategy for sepsis attenuation.

Interestingly, the anti-inflammatory effect of gold derivatives is demonstrated and has been used as an anti-rheumatic drug $[5,6]$. However, gold in these derivatives are in the active oxidizing forms [7] which might be improper to be used in the high oxidative

(c) The Author(s). 2018 Open Access This article is distributed under the terms of the Creative Commons Attribution 4.0 International License (http://creativecommons.org/licenses/by/4.0/), which permits unrestricted use, distribution, and reproduction in any medium, provided you give appropriate credit to the original author(s) and the source, provide a link to the Creative Commons license, and indicate if changes were made. The Creative Commons Public Domain Dedication waiver (http://creativecommons.org/publicdomain/zero/1.0/) applies to the data made available in this article, unless otherwise stated. 
stress condition of sepsis [8-10]. In contrast, gold nanoparticles (AuNP) are inert metallic form with the anti-inflammatory effect. AuNP attenuates macrophage pro-inflammatory cytokine production and spherical AuNP at the diameter of $20-30 \mathrm{~nm}$ is not toxic in either mice or adipose tissue macrophages [7]. AuNP in these diameters are suitable for targeting macrophages by both active and passive mechanisms [11]. It is also interesting to note that the properties and toxicities of AuNP depend on the differences in size and shape, surface modification and molecular conjugations [12, 13]. In addition, the facile production in different sizes and the molecular conjugations of AuNP is relatively un-complicate [7]. Hence, AuNP could be modified for several diverse applications $[14,15]$ and the conjugation of several active substances into AuNP has been studied [16]. However, un-conjugated AuNP demonstrates higher cellular uptake, at least in part, through the higher adsorption of serum proteins on the surface in comparison with the conjugated AuNP [17]. Thus, as a proof of concept for the initial study in sepsis, we selected to test with unconjugated-AuNP. Because macrophage is an important immune cell in sepsis and AuNP reduces pro-inflammatory cytokine production of macrophage, we hypothesize that the proper antibiotics in adjuvant with AuNP might attenuate sepsis severity. Then the effect of AuNP in sepsis was tested in a mouse model of cecal ligation and puncture (CLP) surgery with antibiotic administration. And we also explored if AuNP alter macrophage functions including cytokine production and macrophage polarization.

\section{Results}

\section{Gold nanoparticles (AuNP) attenuate cecal ligation and} puncture sepsis model

Due to the previous demonstration of anti-inflammatory effect of gold nanoparticles (AuNP) [7], we tested whether AuNP altered sepsis-induced mortality, renal dysfunction and liver injury in young outbred ICR mice treated with antibiotics. The anti-inflammatory effect of spherical AuNP in a diameter $21 \mathrm{~nm}$ at the dose of 7.85 $\mu \mathrm{g} /$ gram body weight (approximately $200 \mathrm{ppm}$ per mouse) is previously demonstrated in mice without any toxicities [7]. Hence, AuNP in this dose and at the lower and upper doses were tested with a survival analysis. Interestingly, only AuNP at $7.85 \mu \mathrm{g} / \mathrm{g}$ demonstrated a tendency for sepsis attenuation (Fig. 1a) despite a non-significant value difference. We, then, selected this dose to test further in the sepsis model. Indeed, AuNP that given immediately after CLP surgery attenuated sepsis mortality and reduced blood bacterial burdens, but not bacteria in peritoneal lavage (Fig. 1b-d). AuNP also improved kidney function [evaluated as blood urea nitrogen (BUN) and serum creatinine (Scr)] and liver injury [determined as alanine transaminase (ALT)] at $18 \mathrm{~h}$ post-CLP (Fig. 1e-g). Because cytokine response is one of the important factors in sepsis pathophysiology, proand anti- inflammatory cytokines were evaluated. Mice with AuNP treatment demonstrated the lower level of TNF- $\alpha$, IL-6, IL-1 $\beta$ and IL-10, but not IL-4 (Fig. 1h-1).

Because i) macrophage is important for cytokine activation in sepsis; ii) over pro-inflammatory cytokine production from macrophage leads to cytokine storm in sepsis [18]; and, iii) the balance of pro- (M1) and anti- inflammatory (M2) macrophage polarization might have an impact in sepsis [19], we explored the abundance of M1 and M2 macrophages in the spleens of sepsis mice. There was no difference in the percentage of macrophages $\left(\mathrm{F} 4 / 80^{+}\right)$ in both untreated and AuNP treated sepsis mice. However, AuNP treatment intriguingly showed the reduced percentage of pro-inflammatory macrophage (M1; F4 $/ 80^{+}$and $\mathrm{CD} 86^{+}$) and the increased percentage of anti-inflammatory macrophage (M2; F4/80 ${ }^{+}$ and $\mathrm{CD}_{206}{ }^{+}$) in sepsis as demonstrated by the flow-cytometric analysis (Fig. 2).

\section{Anti-bacterial property and the induction of macrophages} anti-inflammatory responses of gold nanoparticles (AuNP) Although cecal ligation and puncture (CLP) is a sepsis model with poly microbial organisms, gram-negative bacteremia (especially E. coli) is the predominant cause of death in CLP mice [20]. Hence, E. coli was selected to test in our experiments. Interestingly, AuNP demonstrated anti-microbial effect by the reduction of $E$. coli colonies after $4 \mathrm{~h}$ of the incubation (Fig. 3). Although the antibiotic effect of AuNP is weaker than gentamicin antibiotic, this, at least in part, supports the lower blood bacterial burdens in CLP mice with AuNP treatment (Fig. 1c). In addition, effect of AuNP on bone marrow derived macrophages was tested. As such, AuNP attenuated endotoxin-induced macrophage cytokine production as demonstrated by the lower supernatant TNF- $\alpha$ and IL-1 $\beta$ at 6 and $24 \mathrm{~h}$ of the incubation (Fig. 4a, c). AuNP reduced IL- 6 only at $6 \mathrm{~h}$ but not at $24 \mathrm{~h}$ (Fig. $4 \mathrm{~b}$ ) and induced the higher IL-10, an anti-inflammatory cytokine, at 6 and $24 \mathrm{~h}$ of the incubation (Fig. 4d). However, AuNP did not have any effects on IL-4 (Fig. 4e). There was no AuNP dose-related effect on macrophage cytokine production on TNF- $\alpha$, IL-1 $\beta$ and IL-10 at 6 and 24 h of the incubation. But there was a partially dose-related effect on IL-6 at $6 \mathrm{~h}$ of the incubation (Fig. 4). Hence, AuNP reduced several pro-inflammatory cytokines but demonstrated the different effects on anti-inflammatory cytokines (increased IL-10 and no effect on IL-4).

Because high IL-10 production is one of the characteristics of anti-inflammatory macrophage (M2), we tested macrophage polarization with mRNA and protein 


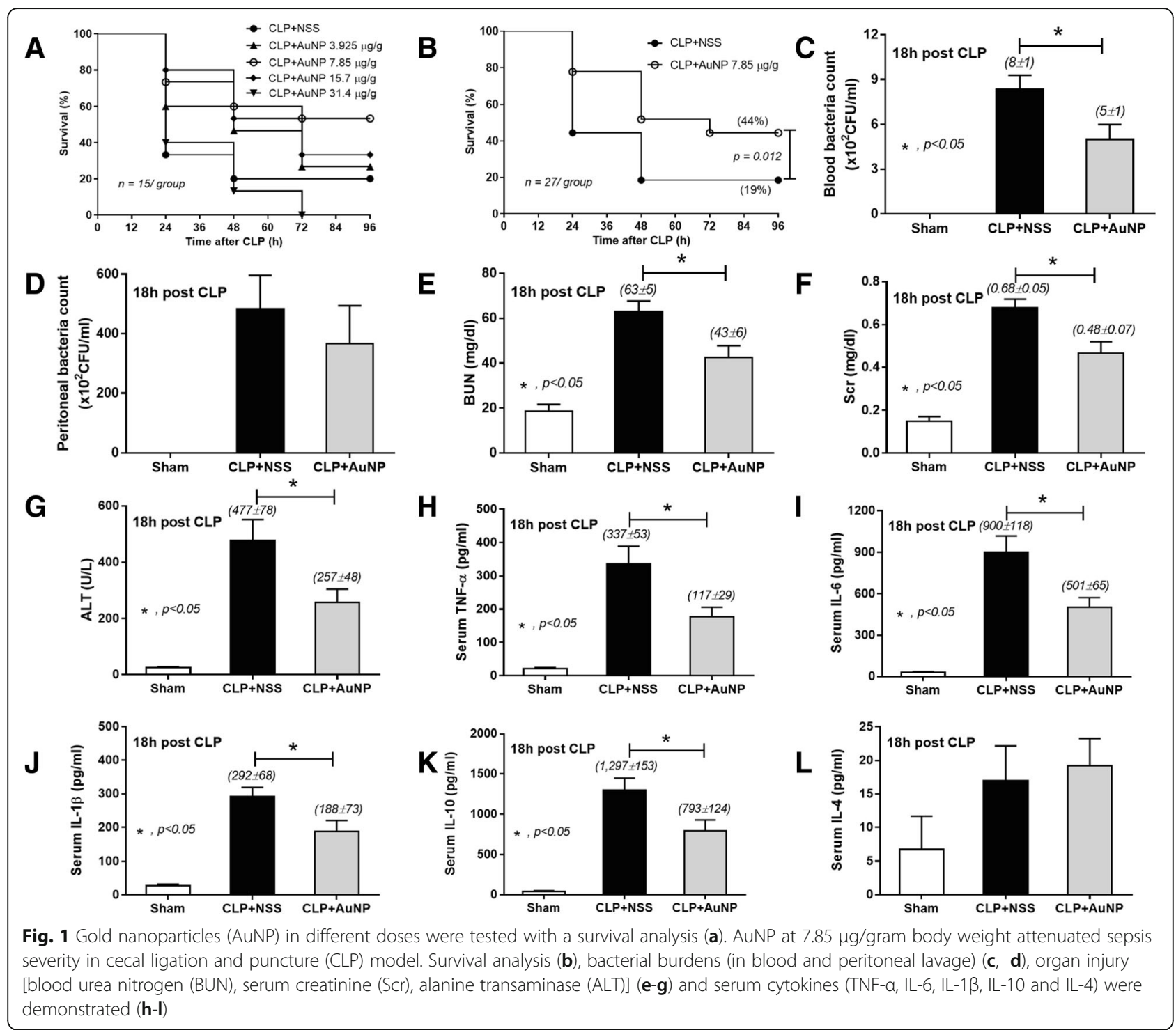

expression. Indeed, the incubation of macrophage with AuNP reduced macrophage production of inducible nitric oxide synthase (iNOS) and increased Arginase 1 (Fig. 5). M1 activation property of AuNP was lower than LPS, a potent M1 polarization stimulator, and not different from IL-4, a negative control of M1 polarization stimulator, as determined by the abundance of Nur77 (Fig. 6a). On the other hand, M2 activation property of AuNP was not different from IL-4, a potent M2 polarization stimulator, as demonstrated by the non-different PPARY activation (Fig. 6b). However, there was no dose-related characteristic in these properties. These data demonstrated the influence of AuNP on the anti-inflammatory functions of macrophages.

\section{Discussion}

The adjuvant effect of spherical gold nanoparticles (AuNP) on antibiotic for the attenuation of bacterial sepsis was demonstrated in a mouse model of polymicrobial sepsis with cecal ligation and puncture (CLP) surgery. AuNP administration, with an appropriate antibiotic, improved sepsis survival through the alteration of macrophage function toward the anti-inflammatory direction.

\section{AuNP attenuated polymicrobial sepsis}

While AuNP in a small diameter are toxic to cells [21], AuNP in a proper range of diameters $(20-30 \mathrm{~nm})$ induce the anti-inflammatory effect [7, 22-24]. In addition, the intravenous administration shows the lowest toxicity in comparison with either oral or intra-peritoneal route [25]. Despite the relatively easy conjugation process of AuNP, un-conjugated AuNP shows higher cellular uptake property [17]. Because sepsis is an imbalance between pro- and anti- inflammation [1] and CLP is a sepsis model of hyperinflammation [26], AuNP might be 

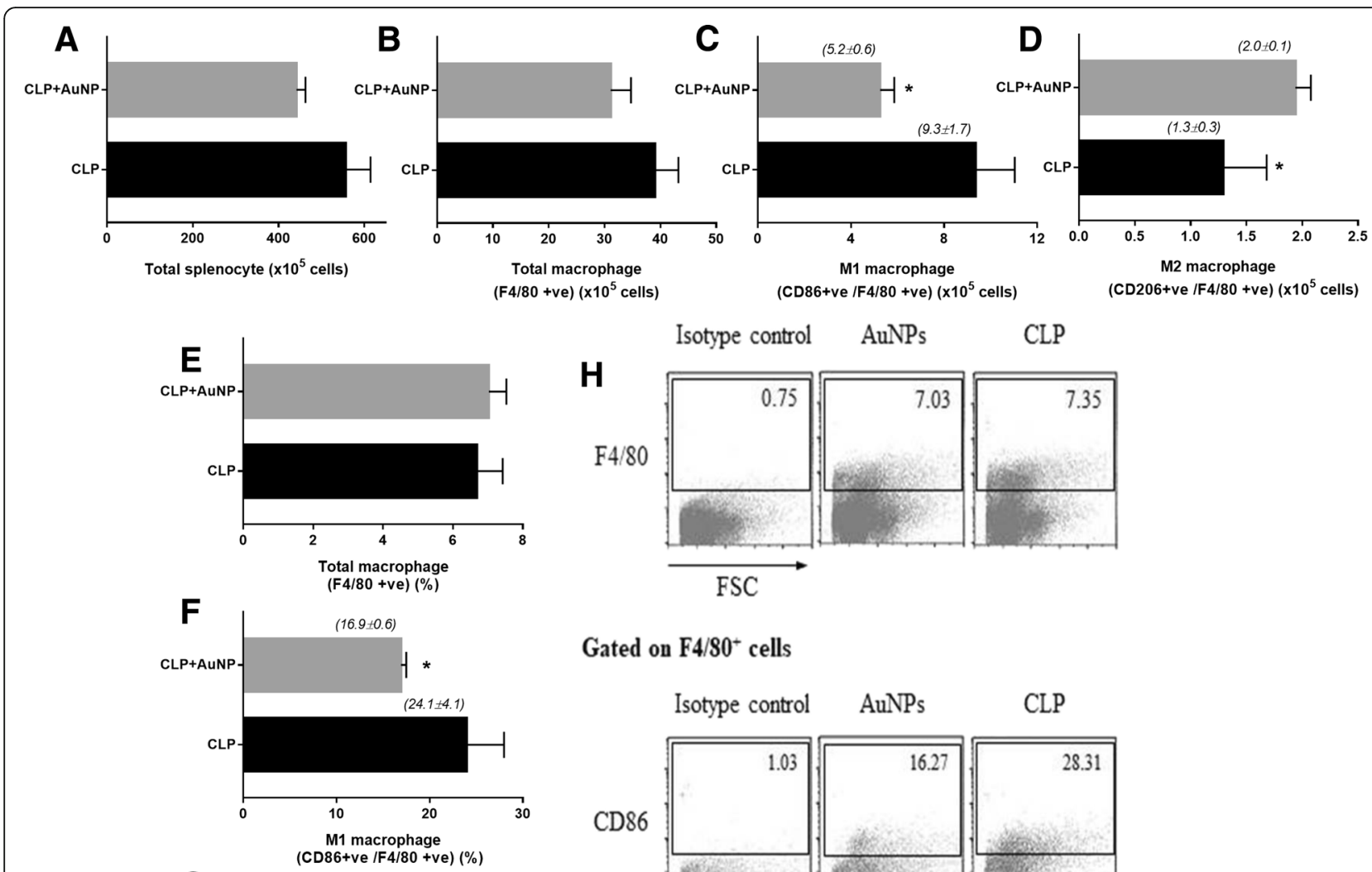

Gated on $\mathrm{F} 4 / 80^{+}$cells

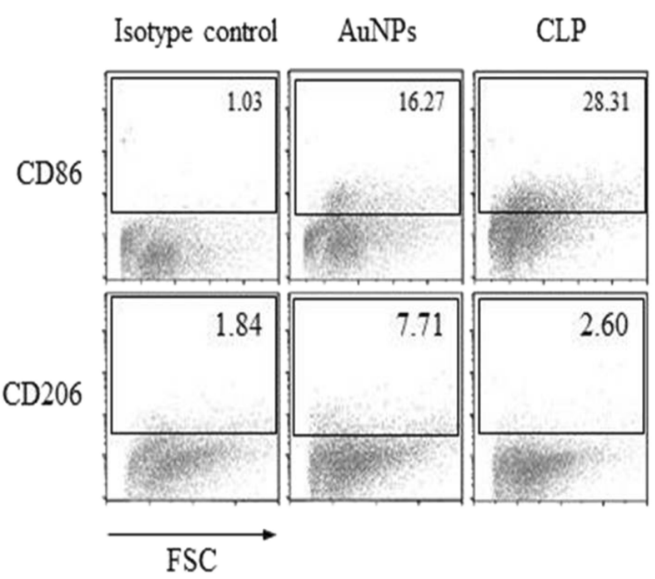

Fig. 2 The quantitative flow-cytometric analysis of spleens from sepsis mice with and without gold nanoparticles (AuNP) administration in the total splenocyte $(\mathbf{a})$, bulk macrophages $\left(\mathrm{F} 4 / 80^{+} ; \mathbf{b}\right)$, macrophages with $\mathrm{M} 1\left(\mathrm{CD} 6^{+}\right.$and $\left.\mathrm{F} 4 / 80^{+} ; \mathbf{c}\right)$ and $\mathrm{M} 2\left(\mathrm{CD} 206^{+}\right.$and $\left.\mathrm{F} 4 / 80^{+} ; \mathbf{d}\right)$ polarization and the percentage of the analysis (e-g) was demonstrated. The representatives of flow-cytometric analysis are shown (h). $\left(n=3-4 /\right.$ group); ${ }^{*}, p<0.05$

beneficial in this model. Hence, we administered AuNP at $21 \mathrm{~nm}$ diameter through the tail vein at post-CLP surgery together with subcutaneously antibiotic administration. Only AuNP at $7.85 \mu \mathrm{g} / \mathrm{g}$, but not the lower and the higher doses, showed a tendency of reduced sepsis mortality rate. The mortality rate of high-dose AuNP, compared with non-treated CLP, was also demonstrated, indicating a possible dose-related toxicity. However, with a proper dose, AuNP attenuated sepsis as measured by mortality, renal dysfunction, liver injury and cytokine levels. Additionally, we also demonstrated a weak antibiotic effect of AuNP supporting a previous publication [27]. The AuNP antibiotic effect might, at least in part, be responsible for the lower blood bacterial burdens.

\section{Anti-inflammatory effect of AuNP}

Due to the rapid natural history of CLP sepsis, the role of macrophage, an important innate immune cells, should be predominant. Interestingly, macrophage polarization is one of the host mechanism to control the proper direction of immune responses; M1 for the pro- and M2 for the anti- inflammatory direction [28]. Indeed, we demonstrated that AuNP decreased pro-inflammatory macrophage (M1) and increased anti-inflammatory macrophage (M2) both in vitro and in vivo, supporting anti-inflammatory effect of AuNP in a previous study [7]. AuNP induced high-Arginase 1 and low-iNOS, the biomarker of M2 and M1 polarization, respectively [29]. Because the predominant IL-10 production is one of the properties of M2 macrophage [28], we tested cytokine respones. As such, AuNP reduced 


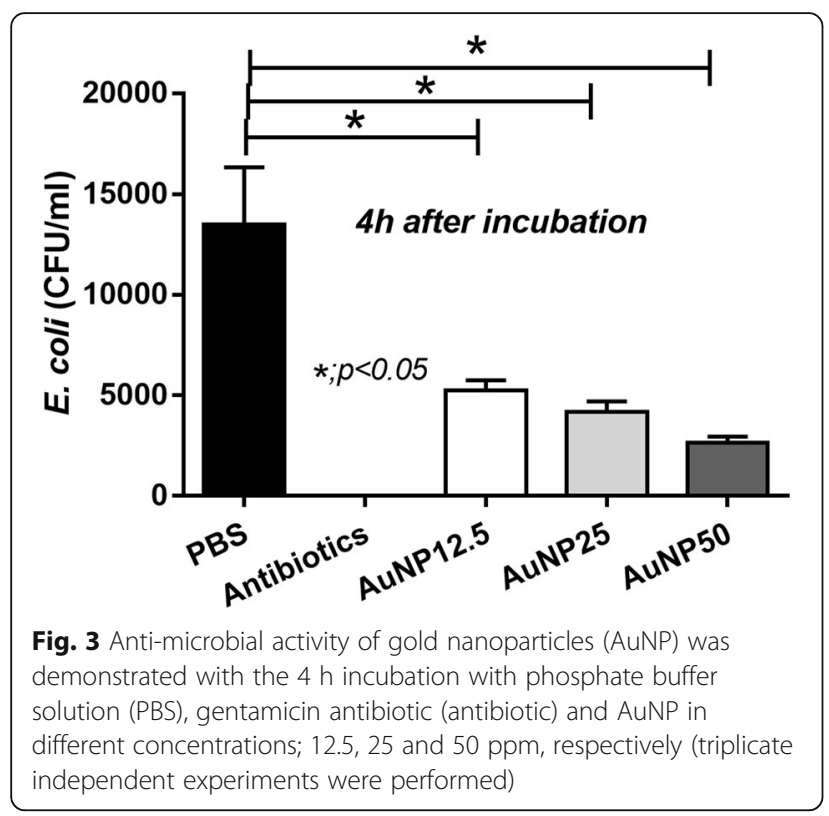

pro-inflammatory cytokines (TNF- $\alpha$, IL- 6 and IL-1 $\beta$ ) but increased anti-inflammatory cytokine (IL-10) in vitro.

It is interesting to note that serum IL-10 in CLP mice with AuNP treatment is not higher than CLP in the control group. This might due to the diversity of AuNP responses in the different cell types. While AuNP predominantly attenuates IL-10 from macrophages, it is possible that AuNP has no effect on other IL-10 producing cells (eg. non-immune cell and lymphocyte) [22]. The response of other cells against AuNP is interesting but out of the scope of our study. On the other hand, the lower serum IL-10 after AuNP treatment might be a balance immune response to the decreased pro-inflammatory cytokines [23]. In addition, AuNP did not show any effects on IL-4, another anti-inflammatory cytokine, both in vivo and in vitro. IL-4 is a well-known predominant cytokine of Th2 cell (immune cell of adaptive immunity) with a lesser influence in innate immune response in sepsis $[30,31]$. Hence, the non-effective IL-4 attenuation of AuNP in our CLP mice implied the lesser impact of AuNP against Th2 in comparison with macrophage [24]. Further studies on this topic will be of great interest.

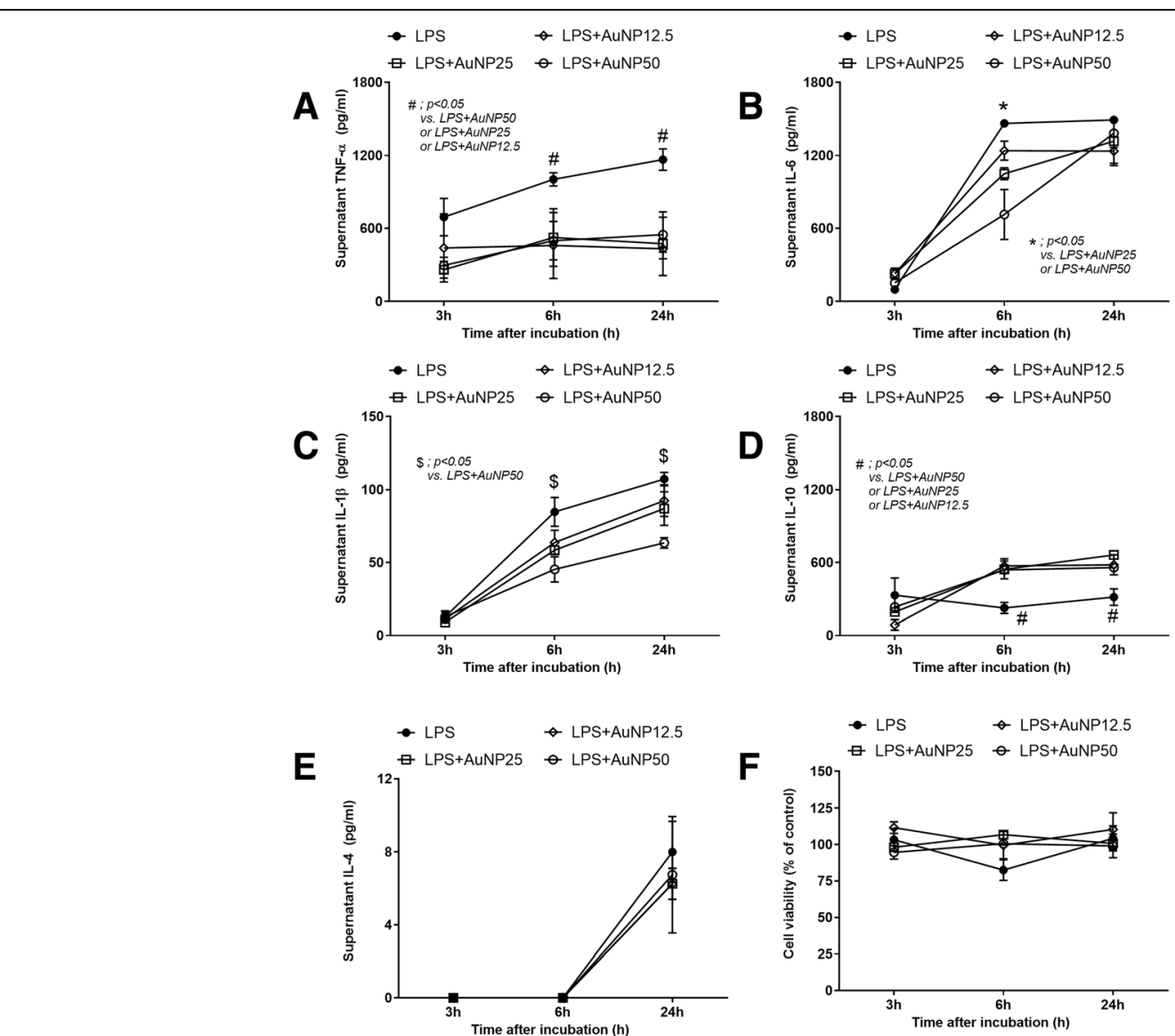

Fig. 4 Macrophage cytokine production of TNF-a (a), IL-6 (b), IL-1 $\beta$ (c), IL-10 (d), IL-4 (e) and cell viability (f) after incubation with endotoxin (LPS) and gold nanoparticles (AuNP) in different concentrations; 12.5, 25 and 50 ppm, respectively, was demonstrated (triplicate independent experiments were performed) 


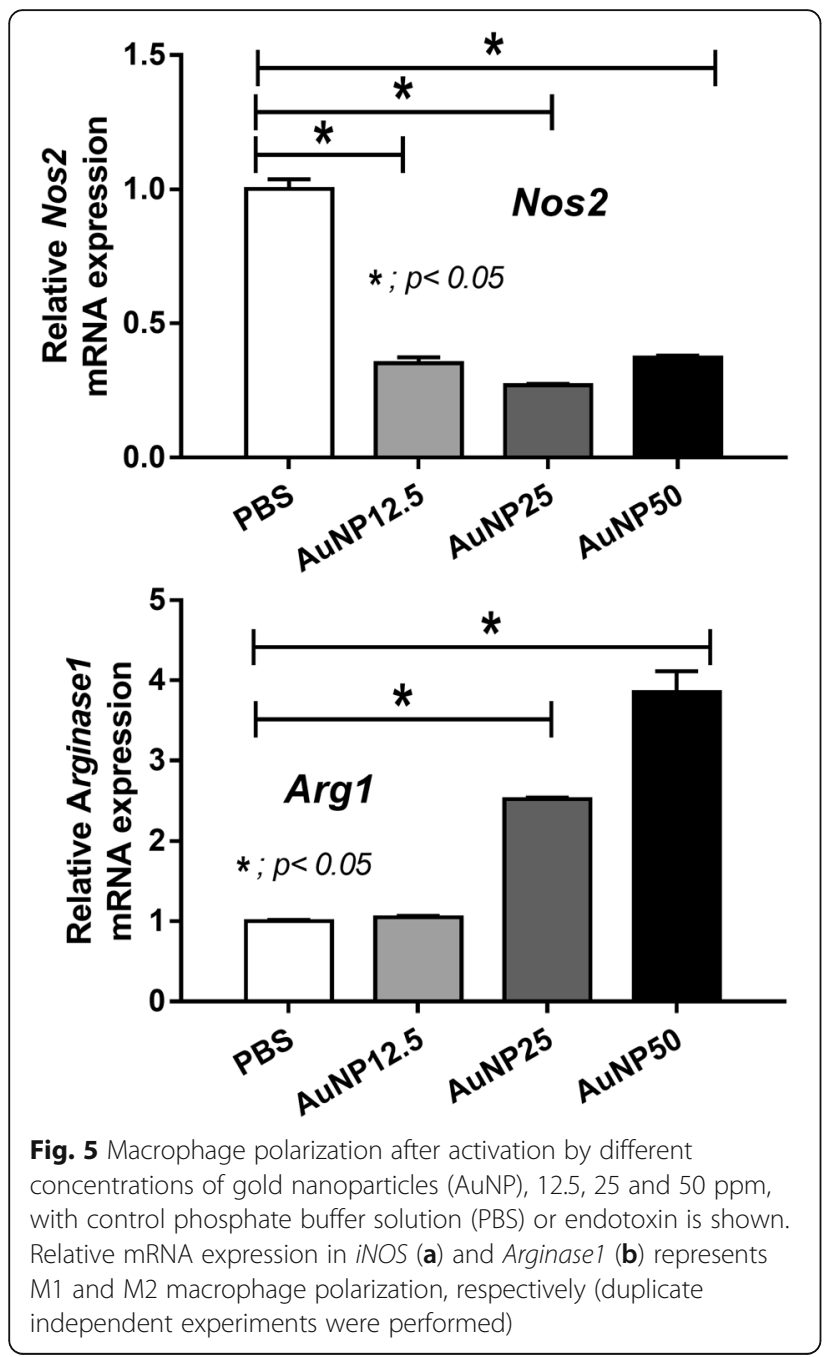

Although the mechanisms of AuNP-induced M2 polarization could not be concluded, nanoparticle size and arginine-binding (-modification) property might be important. As such, the binding activity of metal nanoparticle against amino acid and anti-inflammation of copper nano-particles through arginase modification are mentioned [32]. Perhaps, the proper size of AuNP allows a proper uptake [12, 33], enhances AuNP-arginine binding [34], induces cell stress [35] and arginase modifications leads to M2 polarization. And M2 polarization attenuates polymicrobial sepsis as previously mentioned [36]. More studies are needed to prove this hypothesis. Despite the lack of mechanistic details, our results support the role of AuNP and macrophage manipulation against bacterial sepsis.

\section{Conclusions}

We demonstrated an adjuvant effect of spherical AuNP in $21 \mathrm{~nm}$ diameter on polymicrobial sepsis mouse model. This therapeutic effect was, at least in part, responsible from the induction of macrophage toward anti-inflammatory responses. Although the un-conjugated AuNP was used in our study, the conjugated AuNP with the proper agents might be more beneficial.

\section{Methods}

Gold nanoparticle preparation

Gold nanoparticles were synthesized following the standard citrate-reduction method as previously described $[7,16]$. In brief, $\mathrm{HAuCl}_{4} \cdot 3 \mathrm{H}_{2} \mathrm{O}(1 \mathrm{mM})$ in Milli-Q water with $38.8 \mathrm{mM}$ of sodium citrate was heated at $90{ }^{\circ} \mathrm{C}$. The temperature altered the color of the solution from yellow into deep blue, and shortly afterwards, to deep red. Then the solution in deep red color was heating for another 15 min followed by continuous stirring in room temperature for another $15 \mathrm{~min}$. The final solution containing spherical gold nanoparticles (AuNP) with average size of $21.3 \pm 0.7 \mathrm{~nm}$ was stored at $4{ }^{\circ} \mathrm{C}$, prevented from light until use. Size was confirmed by Ultra-Violet-Visible spectroscopy (Beckman Coulter DU 800 Spectrophotometer, Brea, CA, USA) (data not shown).

\section{Animals and animal models}

The animal procedures followed the US National Institutes of Health (NIH) animal care and use protocols (\#85-23, revised 1985) were implimented. Only male, 8-weeks-old, ICR mice (National Laboratory Animal Center, Nakornpathom, Thailand) were used to avoid the gender difference in sepsis severity [37]. The animal protocols have been approved by the Institutional Animal Care and Use Committee of the Faculty of Medicine, Chulalongkorn University, Bangkok, Thailand.

Cecal ligation and puncture (CLP) procedures followed the previous publications [30]. Briefly, $10 \mathrm{~mm}$ from cecal tip of the large bowel was ligated with silk $2-0$, punctured twice with a 21-gauge needle through an abdominal incision under isoflurane anesthesia. Tramadol (an analgesic drug) at $10 \mathrm{mg} / \mathrm{kg}$ in $0.2 \mathrm{ml}$ of normal saline solution (NSS), for analgesia and imipenem/cilastatin (an antibiotic) at $14 \mathrm{mg} / \mathrm{kg}$ in $0.2 \mathrm{ml}$ of NSS] was administered intraperitoneally and subcutaneously, respectively, at post-operation and at $6 \mathrm{~h}$ later. AuNP diluted in $0.2 \mathrm{ml}$ of NSS were administered through the tail vein at post-operation. AuNP at 3.925, 7.85, 15.7 and $31.4 \mu \mathrm{g} /$ gram body weight were tested with survival experiments in CLP mice and the effective dose $(7.85 \mu \mathrm{g} / \mathrm{g})$ were used in other experiments. Blood was collected through cardiac puncture under isoflurane anesthesia at $18 \mathrm{~h}$-post CLP. Whole blood was used for bacterial burden analysis and the rest of blood was centrifuged at 13,000 $\mathrm{g}$ for $5 \mathrm{~min}$ to separate serum and kept at $-80{ }^{\circ} \mathrm{C}$ until analysis. In the survival study, tramadol and antibiotic were administered once daily for 3 days and until 5 days of observation, respectively. 
A

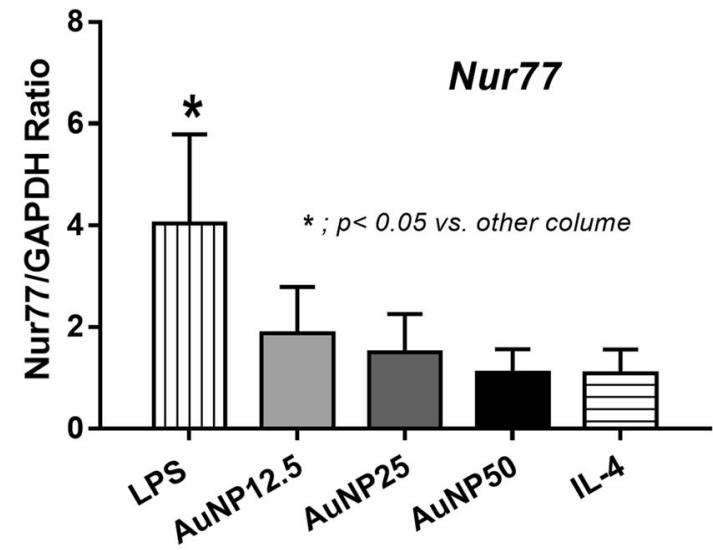

Nur77

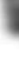

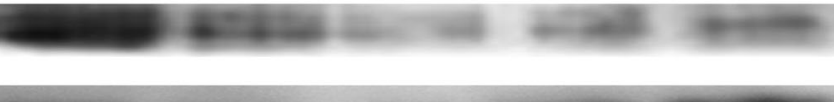

\section{$53 \mathrm{kDa}$}

GAPDH

\section{$37 \mathrm{kDa}$}

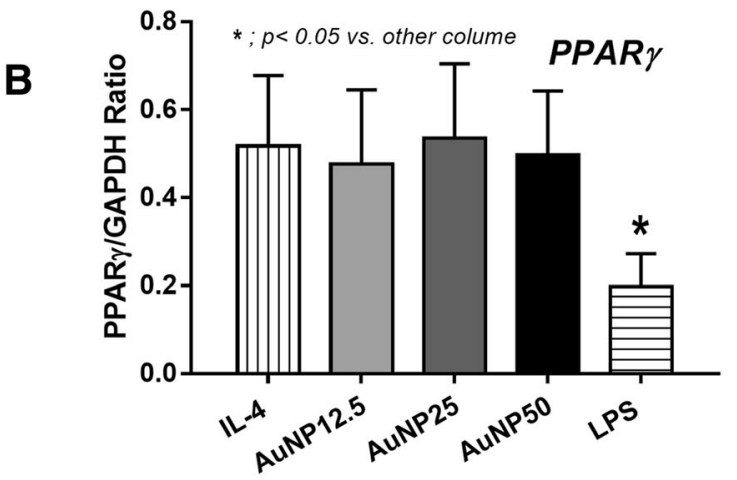

PPAR $\gamma$

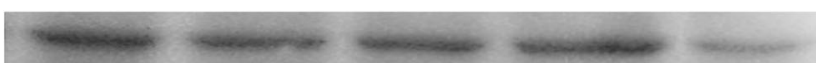

$53 \mathrm{kDa}$

GAPDH

37 kDa

Fig. 6 Macrophage polarization after activation by LPS (for M1 polarization), different concentrations of gold nanoparticles (AuNP; 12.5, 25 and 50 ppm) and IL-4 (for M2 polarization) were performed. The protein expression ratio by Western blot analysis of Nur77 (a) and PPARY (b) represents $\mathrm{M} 1$ and $\mathrm{M} 2$ macrophage polarization, respectively, were demonstrated. And the representatives Western blot analysis are shown (triplicate independent experiments were performed)

\section{Mouse blood sample analysis}

As for quantitative bacterial analysis of blood and peritoneal cavity, $25 \mu \mathrm{l}$ of blood and $1 \mu \mathrm{l}$ of peritoneal fluid from the right para-mesenteric recess, respectively, were spread directly onto blood agar plates (Oxoid, Hampshire, UK), kept at $37{ }^{\circ} \mathrm{C}$ under aerobic conditions, and bacterial colonies were enumerated at $24 \mathrm{~h}$. Serum creatinine (Scr), blood urea nitrogen (BUN) and alanine transaminase (ALT) was measured with QuantiChrom Creatinine Assay (DICT-500; Bioassay, Hayward, CA, USA), QuantiChrome Urea assay (DIUR-500; Bioassay) and EnzyChrom ALT assay (EALT-100, BioAssay). Serum cytokines (TNF- $\alpha$, IL-6, IL-1 $\beta$, IL-10 and IL-4) were measured with ELISA (eBioscience, San Diego, CA,
USA). All assays were performed according to the manufacturer's protocol.

\section{Flow-cytometric analysis of spleen macrophage}

Flow-cytometric analysis was performed following the standard protocol. In brief, spleens were minced in supplemented RPMI-1640 (Roswell Park Memorial Institute media), and the cells were centrifuged at $300 \mathrm{~g}$ for $5 \mathrm{~min}$ at $4{ }^{\circ} \mathrm{C}$. Red blood cells were removed using lysis buffer (Biolegend, CA, USA) and the splenocytes were washed twice in supplemented RPMI-1640. The cells were passed through a cell strainer before cell counting. Subsequently, the splenocytes were block with Fc block (anti-mouse CD16/32 mAbs; Biolegend); then were 
stained with the fluorochrome-conjugated antibodies against mouse F4/80 and CD86 (Biolegend). For intracellular staining, after Fc blocking and F4/80 surface staining, the cells were fixed in $4 \%$ paraformaldehyde overnight. Next, the cells were washed in the intracellular staining buffer and stained with fluorochrome-conjugated antibodies against mouse CD206 (Biolegend). Isotype-matched controls were used as a negative staining. All stained cells were analyzed by flow cytometry (Cytoflex, Beckman Coulter, IN, USA) and the data were analyzed using Kaluza software (Beckman Coulter).

\section{Antibiotics activity of gold nanoparticles}

The procedure for determining antibiotic activity followed a previous publication [27]. Escherichia coli ATCC 25922 (ATCC, Manassas, VA, USA) was sub-cultured in Tryptic soy broth (Thermo Scientific, Waltham, MA, USA) at $37^{\circ}$ $\mathrm{C}$ for $12 \mathrm{~h}$. E. coli approximately at $2.4 \times 10^{6} \mathrm{CFU}$, as determined by a spectrophotometer (ELx808 absorbance reader; BioTek, Shoreline, WA) at optical density $600 \mathrm{~nm}$ at 0.003 (OD $600 \mathrm{~nm}$ at 0.003), were diluted in Tryptic soy broth (Thermo Scientific) and added with AuNP in different concentrations: 12.5, 25 and $50 \mathrm{ppm}$, respectively. Then the solutions were incubated at $37^{\circ} \mathrm{C}$ for $4 \mathrm{~h}$. After that, the supernatant in serial dilution was plated in Tryptic soy agar (Thermo Scientific), kept at $37{ }^{\circ} \mathrm{C}$ overnight before bacterial colony enumeration. E. coli in Tryptic soy broth alone or in $100 \mu \mathrm{g} / \mathrm{ml}$ of gentamicin were used as the positive and negative control group, respectively.

\section{Bone marrow derived macrophage preparation}

Macrophages were derived from bone marrow (BM) following the previous published methods $[3,31]$ and bone marrow from femurs were obtained following the previous published methods [38]. In brief, BM cells were obtained from femurs by sacrificed mice under euthanize condition and open the abdominal cavity to find the pelvic-hip joint. Then cut off the hind leg above the pelvic-hip joint and cut off the tibia at below the knee joint. Remove the muscles and tissues around the femur with sterile forceps, scissors and gauze. After that, centrifuge at $6000 \mathrm{rpm}$ for $4{ }^{\circ} \mathrm{C}$ to collect cells from bone marrow and incubated for 7 days with Dulbecco's Modified Eagle Medium (DMEM) supplemented with $10 \%$ fetal bovine serum (FBS), $1 \%$ penicillin/streptomycin, 4-(2-hydroxyethyl)-1-piperazineethanesulfonic acid (HEPES) with sodium pyruvate in a humidified $5 \% \mathrm{CO}_{2}$ incubator at $37{ }^{\circ} \mathrm{C}$. Conditioned media of the L929 cell line, containing macrophage-colony stimulating factor, at $20 \% w / v$ were used to induce macrophages from BM-derived pleuripotent stem cells. Cells were harvested with cold phosphate buffer solution (PBS) and the macrophage phenotype was analyzed with anti-F4/80 and
anti-CD11c antibody staining (BioLegend, San Diego, CA, USA) by flow cytometry (data not shown).

\section{Induction of macrophage cytokine production protocol} BM-derived macrophages at $1 \times 10^{5}$ cells/well were plated in 96-well tissue culture plate, then incubated with $100 \mathrm{ng} /$ ml of LPS of Escherichia coli 026:B6 (Sigma-Aldrich, St. Louis, MO, USA) and different concentration of AuNP: 12.5, 25 and $50 \mathrm{ppm}$, respectively, in $5 \% \mathrm{CO}_{2}$ incubator at $37{ }^{\circ} \mathrm{C}$ for 3, 6 and $24 \mathrm{~h}$. AuNP was adjusted into the similar volume for the incubation. At each time point, the culture supernatant was collected and stored at $-80{ }^{\circ} \mathrm{C}$ until analysis. Then the supernatant was measured for cytokines (TNF- $\alpha$, IL-6, IL-1 $\beta$, IL-10 and IL-4) with ELISAs assay (eBioscience, San Diego, CA, USA) followed the manufacturer's instructions. In addition, macrophage viability was measured after the incubation by the MTS assay (One Solution Cell Proliferation Assay, Promega Corporation, Madison, Wis, USA) according to the manufacturer's instructions. Cell viability at each time point in the different conditions was calculated into percentage of cell viability with PBS control.

\section{Analysis of macrophage polarization}

The protocol has been adapted from a previously published method [29, 32]. In brief, BM-derived macrophages $5 \times 10^{5}$ cells/well were plated in 12-well tissue culture plate. Cells were activated into M1 and M2 with $100 \mathrm{ng} / \mathrm{ml}$ of LPS (Sigma-Aldrich) and $10 \mathrm{ng} / \mathrm{ml}$ of IL-4 (Sigma-Aldrich), respectively, with the presentation of AuNP in the different concentrations (12.5, 25 and $50 \mathrm{ppm})$ or PBS control. All groups were incubated in $5 \% \mathrm{CO}_{2}$ incubator at $37{ }^{\circ} \mathrm{C}$ for $3 \mathrm{~h}$, then the culture supernatant was removed and $400 \mathrm{ul}$ of TRIzol reagent (Invitrogen, Carlsbad, CA, USA) was added per well to extract RNA from the macrophages followed to the manufacturer's protocol. The purified RNA was synthesized into complementary DNA, amplified by quantitative polymerase chain reaction (qPCR) by used of iNOS and Arginase 1 for characterize of M1 and M2 macrophages, respectively. The nucleotide sequences were as followed: iNOS, forward, 5'-CCCTTCCGAAGTTTCTG GCAGCAGC-3',reverse, 5'-GGCTGTCAGAGCCTCGT GGCTTT G-3'; Arginase1, forward, 5'CAGAAGAATG GAAGAGTCAG-3',reverse: 5'-CAGATATGCA GGGA GTCACC-3'. The expression of each gene was normalized to the expression of $\beta$-actin by the $2-\Delta \Delta \mathrm{CT}$ method.

In addition, macrophage polarization was supported by Western blot analysis of Nur77 and PPAR $\gamma$ for M1 and M2 characterizations, respectively. In brief, macrophages $1 \times 10^{6}$ cells/well were plated in a 12-well tissue culture plate with the conditions of LPS, IL-4 and AuNP. The cell harvest procedures were performed as mentioned above. Then the cells were added with RIPA lysis 
and extraction buffer, sonicated, centrifuged $10 \mathrm{~min}$ at $1500 \mathrm{rpm}, 4{ }^{\circ} \mathrm{C}$ for the supernatant collection. Protein concentrations were determined by the bicinchoninic acid (BCA) assay and $20 \mu \mathrm{g}$ proteins were separated by SDS-PAGE and transfer to Polyvinylidene difluoride (PVDF) membrane (activated by 100\% methanol). The membrane was blocked with $5 \%$ bovine serum albumin (BSA) in tris-buffered saline with tween 20 solution, then incubated at $4{ }^{\circ} \mathrm{C}$ overnight with anti-Nur77 (from Barbara Osborne, MA, USA) or anti-PPARy (Cell Signaling Technology, Denvers, MA, USA) and anti-GAPDH (Santa Cruz Biotechnology, Dallas, TX, USA). The chemiluminescence bands were detected by the chemiluminescence detector (ImageQuant LAS 4000, GE Healthcare Life Sciences, Chicago, IL, USA).

\section{Statistical analysis}

Data were analyzed as mean \pm standard error (SE); the differences between groups were examined for statistical significance by one-way analysis of variance (ANOVA) followed by Bonferroni analysis for the experiments with multiple time-point data collection. The survival analysis was determined by log-rank test. The repeated measures analysis of variance (ANOVA) with Bonferroni post hoc analysis was used for the analysis of the time-course experiments. All statistical analyses were performed with SPSS 11.5 software (SPSS, IL, USA). $P$ value $<0.05$ was considered statistically significant.

\section{Abbreviations \\ ALT: Alanine transaminase; ANOVA: Analysis of variance; AuNP: Gold nanoparticles; BCA: Bicinchoninic acid; BM: Bone marrow; BSA: Bovine serum albumin; BUN: Blood urea nitrogen; CLP: Cecal ligation and puncture; DMEM: Dulbecco's Modified Eagle Medium; ELISA: Enzyme linked immunosorbent assays; FBS: Fetal bovine serum; HEPES: 4-(2-hydroxyethyl)-1- piperazineethanesulfonic acid; IL-10: Interleukin 10; IL-1 $\beta$ : Interleukin 1 beta; IL-4: Interleukin 4; IL-6: Interleukin 6; iNOS: inducible nitric oxide synthase; NSS: Normal saline solution; PBS: Phosphate buffer solution; \\ PVDF: Polyvinylidene difluoride; GPCR: Quantitative polymerase chain reaction; Scr: Serum creatinine; SE: Standard error; TNF-a: Tumor necrosis factor-alpha}

\section{Acknowledgements \\ AL worked under Center of Excellence in Immunology and Immune-mediated Diseases, Department of Microbiology, Faculty of Medicine. We also thank Oral Biology Research Center, Faculty of Dentistry, Chulalongkorn University for Flow cytometry facility. \\ Funding \\ This study was funded by Thailand Government Fund 2017 (RSA60) and Ratchadaphiseksomphot Endowment Fund 2017 (76001-HR) Chulalongkorn University. ST was funded by the Scholarship from the Graduate School, Chulalongkorn University to commemorate the $72^{\text {nd }}$ anniversary of his Majesty King Bhumibol Adulyadej and the $90^{\text {th }}$ Anniversary Chulalongkorn University Fund (Ratchadaphiseksomphot Endowment Fund). The funding body had no role in the design of the study and collection, analysis, and interpretation of data at the same in writing the manuscript.}

\section{Availability of data and materials}

The datasets generated during and/or analysed during the current study are available from the corresponding author on reasonable request.

\section{Disclosure statement}

This study was funded by Thailand Government Fund 2017 (RSA60) and Ratchadaphiseksomphot Endowment Fund 2017 (76001-HR) Chulalongkorn University. ST was funded by the Scholarship from the Graduate School, Chulalongkorn University to commemorate the $72^{\text {nd }}$ anniversary of his Majesty King Bhumibol Adulyadej and the $90^{\text {th }}$ Anniversary Chulalongkorn University Fund (Ratchadaphiseksomphot Endowment Fund).

\section{Authors' contributions}

AL and ST designed the study and drafted the final manuscript. AL, ST, TO and SS performed the main experiments. NS and CTBV performed GPCR and flow cytometry. AS participated in gold nanoparticles research. TP and PR participated in M1 M2 research. All authors finally read and approved the final manuscript to be published.

\section{Ethics approval and consent to participate}

The animal protocols were approved by the Institutional Animal Care and Use Committee of the Faculty of Medicine, Chulalongkorn University, Bangkok, Thailand.

\section{Consent for publication}

Not applicable.

\section{Competing interests}

The authors declare that they have no competing interests.

\section{Publisher's Note}

Springer Nature remains neutral with regard to jurisdictional claims in published maps and institutional affiliations.

\section{Author details}

${ }^{1}$ Medical Microbiology, Interdisciplinary Program, Graduate School, Chulalongkorn University, Bangkok, Thailand. ${ }^{2}$ Department of Microbiology, Faculty of Science, Chulalongkorn University, Bangkok, Thailand. ${ }^{3}$ Oral Biology program, Faculty of Dentistry, Chulalongkorn University, Bangkok, Thailand. ${ }^{4}$ Medical Sciences Program, Faculty of Medicine, Chulalongkorn University, Bangkok, Thailand. ${ }^{5}$ Department of Anatomy, Faculty of Medicine, Chulalongkorn University, Bangkok, Thailand. ${ }^{6}$ Department of Microbiology and Immunology and Research Unit of Oral Microbiology, Chulalongkorn University, Bangkok, Thailand. 'Department of Microbiology, Faculty of Medicine, Chulalongkorn University, Bangkok 10330, Thailand. ${ }^{8}$ Center of Excellence in Immunology and Immune-mediated Diseases, Department of Microbiology, Faculty of Medicine Chulalongkorn University, Bangkok, Thailand.

Received: 8 January 2018 Accepted: 6 August 2018

Published online: 17 August 2018

\section{References}

1. Singer M, Deutschman CS, Seymour CW, Shankar-Hari M, Annane D, Bauer $M$, et al. The third international consensus definitions for Sepsis and septic shock (Sepsis-3). JAMA. 2016;315(8):801-10.

2. Doi K, Leelahavanichkul A, Yuen PST, Star RA. Animal models of sepsis and sepsis-induced kidney injury. J Clin Invest. 2009;119(10):2868-78.

3. Ondee T, Surawut S, Taratummarat S, Hirankan N, Palaga T, Pisitkun P, et al. FC Gamma Receptor IIB Deficient Mice: A Lupus Model with Increased Endotoxin Tolerance-Related Sepsis Susceptibility. Shock. 2017;47(6):743-752.

4. Hotchkiss RS, Monneret G, Payen D. Immunosuppression in sepsis: a novel understanding of the disorder and a new therapeutic approach. Lancet Infect Dis. 2013;13(3):260-8.

5. Kean WF, Kean IRL. Clinical pharmacology of gold. Inflammopharmacology. 2008;16(3):112-25.

6. Ujfalussy I, Koó É, Seszták M, Gergely P. Termination of disease-modifying antirheumatic drugs in rheumatoid arthritis and in psoriatic arthritis. $Z$ Rheumatol. 2003;62(2):155-60.

7. Chen H, Dorrigan A, Saad S, Hare DJ, Cortie MB, Valenzuela SM. In vivo study of spherical gold nanoparticles: inflammatory effects and distribution in mice. PLoS One. 2013;8(2):e58208.

8. Alobaidi R, Basu RK, Goldstein SL, Bagshaw SM. Sepsis-associated acute kidney injury. Semin Nephrol. 2015;35(1):2-11. 
9. Arulkumaran N, Deutschman CS, Pinsky MR, Zuckerbraun B, Schumacker PT, Gomez H, et al. MITOCHONDRIAL FUNCTION IN SEPSIS. Shock (Augusta, Ga). 2016;45(3):271-81.

10. Draganov D, Teiber J, Watson C, Bisgaier C, Nemzek J, Remick D, et al. PON1 and oxidative stress in human sepsis and an animal model of sepsis. Adv Exp Med Biol. 2010;660:89-97.

11. Pissuwan D, Valenzuela SM, Killingsworth MC, Xu X, Cortie MB. Targeted destruction of murine macrophage cells with bioconjugated gold nanorods. J Nanopart Res. 2007;9(6):1109-24.

12. Chithrani BD, Ghazani AA, Chan WC. Determining the size and shape dependence of gold nanoparticle uptake into mammalian cells. Nano Lett. 2006;6(4):662-8.

13. Fischer HC, Chan WC. Nanotoxicity: the growing need for in vivo study. Curr Opin Biotechnol. 2007;18(6):565-71.

14. Dykman LA, Khlebtsov NG. Gold nanoparticles in biology and medicine: recent advances and prospects. Acta Nat. 2011;3(2):34-55.

15. Mieszawska AJ, Mulder WJM, Fayad ZA, Cormode DP. Multifunctional gold nanoparticles for diagnosis and therapy of disease. Mol Pharm. 2013;10(3):831-47.

16. Pissuwan D, Cortie C, Valenzuela S, Cortie M. Gold nanosphere-antibody conjugates for hyperthermal therapeutic applications. Gold Bull. 2007; 40(2):121-9.

17. Chithrani BD, Chan WC. Elucidating the mechanism of cellular uptake and removal of protein-coated gold nanoparticles of different sizes and shapes. Nano Lett. 2007;7(6):1542-50

18. Cavaillon JM, Adib-Conquy M. Monocytes/macrophages and sepsis. Crit Care Med. 2005;33(12 Suppl):S506-9.

19. Ka MB, Daumas A, Textoris J, Mege JL. Phenotypic diversity and emerging new tools to study macrophage activation in bacterial infectious diseases. Front Immunol. 2014;5:500.

20. Hyde SR, Stith RD, McCallum RE. Mortality and bacteriology of sepsis following cecal ligation and puncture in aged mice. Infect Immun. 1990; 58(3):619-24.

21. Brandenberger C, Rothen-Rutishauser B, Mühlfeld C, Schmid O, Ferron GA Maier $\mathrm{KL}$, et al. Effects and uptake of gold nanoparticles deposited at the air-liquid interface of a human epithelial airway model. Toxicol Appl Pharmacol. 2010;242(1):56-65.

22. Shaw DM, Merien F, Braakhuis A, Dulson D. T-cells and their cytokine production: the anti-inflammatory and immunosuppressive effects of strenuous exercise. Cytokine. 2017;

23. Hotchkiss RS, Monneret G, Payen D. Sepsis-induced immunosuppression: from cellular dysfunctions to immunotherapy. Nat Rev Immunol. 2013; 13(12):862-74

24. Edholm ES, Rhoo KH, Robert J. Evolutionary aspects of macrophages polarization. Results Probl Cell Differ. 2017;62:3-22.

25. Zhang XD, Wu HY, Wu D, Wang YY, Chang JH, Zhai ZB, et al. Toxicologic effects of gold nanoparticles in vivo by different administration routes. Int J Nanomedicine. 2010;5:771-81.

26. Doi K, Hu X, Yuen PST, Leelahavanichkul A, Yasuda H, Kim SM, et al. AP214, an analogue of a-melanocyte-stimulating hormone, ameliorates sepsisinduced acute kidney injury and mortality. Kidney Int. 2008;73(11):1266-74.

27. Zhou Y, Kong Y, Kundu S, Cirillo JD, Liang H. Antibacterial activities of gold and silver nanoparticles against Escherichia coli and bacillus CalmetteGuerin. J Nanobiotechnology. 2012;10:19.

28. Kimura T, Nada S, Takegahara N, Okuno T, Nojima S, Kang S, et al. Polarization of M2 macrophages requires Lamtor1 that integrates cytokine and amino-acid signals. Nat Commun. 2016;7:13130.

29. Jablonski KA, Amici SA, Webb LM, JdD R-R, Popovich PG, Partida-Sanchez S, et al. Novel Markers to Delineate Murine M1 and M2 Macrophages. PLOS ONE. 2015;10(12):e0145342.

30. Leelahavanichkul A, Souza ACP, Street JM, Hsu V, Tsuji T, Doi K, et al. Comparison of serum creatinine and serum cystatin $C$ as biomarkers to detect sepsis-induced acute kidney injury and to predict mortality in CD-1 mice. Am J Physiol Ren Physiol. 2014;307(8):F939-F48.

31. Surawut S, Ondee T, Taratummarat S, Palaga T, Pisitkun P, Chindamporn A et al. The role of macrophages in the susceptibility of fc gamma receptor Ilb deficient mice to Cryptococcus neoformans. Sci Rep. 2017;7:40006.

32. Wongchana W, Lawlor RG, Osborne BA, Palaga T. Impact of Notch1 deletion in macrophages on Proinflammatory cytokine production and the outcome of experimental autoimmune encephalomyelitis. J Immunol. 2015;195(11):5337-46.
33. Albanese A, Tang PS, Chan WC. The effect of nanoparticle size, shape, and surface chemistry on biological systems. Annu Rev Biomed Eng. 2012;14:1-16

34. Tomoaia G, Frangopol PT, Horovitz O, Bobos LD, Mocanu A, Tomoaia-Cotisel $M$. The effect of arginine on gold nanoparticles in colloidal solutions and in thin films. J Nanosci Nanotechnol. 2011;11(9):7762-70.

35. Shan B, Wang X, Wu Y, Xu C, Xia Z, Dai J, et al. The metabolic ER stress sensor IRE1alpha suppresses alternative activation of macrophages and impairs energy expenditure in obesity. Nat Immunol. 2017;18(5):519-29.

36. Nemeth K, Leelahavanichkul A, Yuen PS, Mayer B, Parmelee A, Doi K, et al. Bone marrow stromal cells attenuate sepsis via prostaglandin E(2)dependent reprogramming of host macrophages to increase their interleukin-10 production. Nat Med. 2009;15(1):42-9.

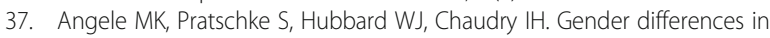
sepsis: cardiovascular and immunological aspects. Virulence. 2014:5(1):12-9.

38. Liu X, Quan N. Immune cell isolation from mouse femur bone marrow. Bioprotocol. 2015:5(20):e1631.
Ready to submit your research? Choose BMC and benefit from:

- fast, convenient online submission

- thorough peer review by experienced researchers in your field

- rapid publication on acceptance

- support for research data, including large and complex data types

- gold Open Access which fosters wider collaboration and increased citations

- maximum visibility for your research: over $100 \mathrm{M}$ website views per year

At BMC, research is always in progress.

Learn more biomedcentral.com/submissions 